\title{
OBSERVATION EQUIVALENCES FOR THE SEMANTICS OF INHERITANCE
}

\author{
Claudia Balzarotti, Fiorella De Cindio, Lucia Pomello \\ Dipartimento di Scienze dell'Informazione, Università di Milano \\ via Comelico 39 - 20135 Milano (Italy) \\ e-mail:decindio@dsi.unimi.it,pomello@dsi.unimi.it
}

\begin{abstract}
In the object-oriented languages, inheritance is a fundamental relation among classes, that originally indicated the $i s-a$ relation; however it has been often used for code-reuse. The integration between object-orientation and concurrency gives the possibility of using theoretical tools, such as the notions of action and state observation equivalences and preorders, developed within concurrency theories (Petri nets, CCS). In this paper we propose a semantic characterisation of two forms of inheritance in concurrent context: in particular, we propose a preorder based on action observation to give the semantics of inheritance used as is-a relation (type inheritance), and a preorder based on state observation for the semantics of inheritance as code-reuse (implementation inheritance).
\end{abstract}

Keywords: object-orientation, concurrency, inheritance, Petri nets, observation equivalences.

\section{Introduction}

The main entity of the object-oriented languages is the object, which is generally defined by a class. A fundamental relation between classes is inheritance, that originally indicated the is- $a$ relation among classes. On the other hand, the success of object-oriented programming languages is due to the use of inheritance for code-reuse. Inheritance is often used in these two different ways. The aim of this paper is studying these two different faces of the inheritance relation in concurrent object-oriented languages. The unclear distinction between these two different interpretations of the notion of inheritance is misleading in object oriented software development and, as far as we know, does not find solution in the literature.

The integration of object-orientation with concurrency, yielding systems of active 
objects modelled as concurrent processes, gives the possibility of using theoretical tools, such as the notions of observation preorders and equivalences, for the study of the inheritance relation. In particular, the place-transition duality in Petri nets [9] allows the definition of two types of preorders and equivalences between nets, based on action observation and on state observation [13]. A variety of observation preorders and equivalences have been already used for defining the semantics of the inheritance relation. Among the various proposals in the literature (see for a survey [12], [2]), we take into consideration those proposed by Nierstrasz [11] and van der Aalst and Basten [1] for what concerns action observability, while we consider the approach of CLOWN [5] for what concerns state observability.

Nierstrasz's hypothesis is that the sequences of requests that an object can accept constitute a regular language. Moreover, Nierstrasz defines a preorder based on failures, the Request Substitutability preorder (RS-preorder, $s_{\mathrm{RS}}$ ), and considers this preorder as the semantics of the subtyping relation according to Wegner and Zdonik's substitutability principle [15]. Van der Aalst and Basten introduce preorders too, based on the branching bisimulation equivalence, and two operators, the encapsulation operator $\delta_{\mathrm{H}}$ and the abstraction operator $\tau_{\mathrm{I}}$, that removes and respectively labels as not observable, the transitions corresponding to the methods of the subclass not inherited from the superclass.

CLOWN is a concurrent object-oriented specification language whose basic building block is the elementary class, which specifies sequential objects through a set of instance data and methods, a typed interface and its (multiple) inheritance link(s). A labelled net associated with each elementary class describes the casual relationship between methods execution. The definition of the inheritance relation between classes is based on the State Transformation preorder between the corresponding nets. The State Transformation preorder (ST-preorder, $s_{\mathrm{ST}}$ ) [14] compares systems with respect to their state space.

In this paper we develop the proposal sketched in [7] and propose a semantic characterisation of two forms of inheritance in concurrent contexts, based on two preorders: preorders are better suited than equivalence notions for modelling the behaviour extension.

According to the terminology used in [10], we use the term type inheritance to denote subtyping, i.e. the is-a relation among classes, and the term implementation inheritance to denote code-reuse. According to [3], type inheritance concerns and compares the external observable behaviour of objects; implementation inheritance does the same with respect to their internal structure. As a consequence, type inheritance will be based on a new action-based preorder resulting from an original integration of Nierstrasz's and van der Aalst and Basten's proposals. Implementation inheritance will be based on the State Transformation preorder.

We omit here to deal with instance data, (typed) interfaces, object creation and other topics which are typical issues for the design of a concurrent object-oriented specification language, and have been partially addressed in CLOWN. As usable formalisms require sound notions, here we make a step backward and, according to Nierstrasz, focus on basic behaviour.

The paper is organized as follows: in the section "basic definitions", we give some basic definitions about the notions based on action and state observation, in the section "examples and open problems" we discuss the inheritance relations proposed in the literature through examples, in the section "the preorders based on action observability" we define new preorders based on action observability, in the section 
"soundness of the preorders based on action observability" we prove the soundness of these preorders, while in the section "inheritance as subtyping and code reuse" we give the definitions of type inheritance and implementation inheritance and show their use on the examples introduced in the section "examples and open problems".

\section{Basic definitions}

We consider Elementary Net (EN) systems [9], the basic class of Petri Nets. Formally, a net is a 3-tuple $(\mathrm{B}, \mathrm{E}, \mathrm{F})$, where $\mathrm{B}$ is a finite set of places, $\mathrm{E}$ is a finite set of transitions such that $\mathrm{B} \cap \mathrm{E}=\varnothing$ and $\mathrm{B} \cup \mathrm{E} \neq \varnothing$, and $\mathrm{F} \subseteq(\mathrm{B} \times \mathrm{E}) \cup(\mathrm{E} \times \mathrm{B})$ is a transition function such that $\underset{\operatorname{dom}}{(F)} \cup \underline{\operatorname{ran}}(\mathrm{F})=\mathrm{B} \cup \mathrm{E}$. Given $\mathrm{x} \in \mathrm{X}=\mathrm{B} \cup \mathrm{E}$, the pre-set of $x$ is the set $\bullet x=\{y \in X:(y, x) \in F\}$ and the post-set of $x$ is the set $x^{\bullet}=\{y$ $\in X:(x, y) \in F\}$.

An EN systems is a 4-tuple (B,E,F, $\left.c_{i n}\right)$, where $(B, E, F)$ is a net and $c_{i n} \subseteq B$ is the initial case. Given an $\mathrm{EN}$ system $\Sigma=\left(\mathrm{B}, \mathrm{E}, \mathrm{F}, \mathrm{c}_{\mathrm{in}}\right), \mathrm{e} \in \mathrm{E}$ is enabled at the case $\mathrm{c} \subseteq \mathrm{B}$, denoted $\mathrm{cle>}$, iff $\bullet \mathrm{e} \subseteq \mathrm{c}$; if $\mathrm{e}$ is enabled at $\mathrm{c}$, then the occurrence of e leads from $\mathrm{c}$ to a case $c^{\prime}$, denoted $c \mid e>c^{\prime}$, iff $c \mid e>$ and $c^{\prime}=(c-\bullet e) \cup e^{\bullet}$. Given $w=e_{1} e_{2} \ldots e_{n} \in E^{*}, w$ is enabled at the case $c \subseteq B$, denoted $c \mid w>$, iff $\exists c_{1}, c_{2}, \ldots, c_{n-1}$ such that $c\left|e_{1}>c_{1}\right| e_{2}>c_{2} \ldots$ $c_{n-1} \mid e_{n}>$; the occurrence of $w$ leads from $c$ to $c^{\prime}$, denoted $c \mid w>c^{\prime}$, iff $c\left|e_{1}>c_{1}\right| e_{2}>c_{2} \ldots$ $c_{n-1} \mid e_{n}>c^{\prime}$. The set of cases, denoted $C_{\Sigma}$, is the smallest subset of $2^{B}$ such that $c_{i n} \subseteq$ $\mathrm{C}_{\Sigma}$, and $\forall \mathrm{c} \in \mathrm{C}_{\Sigma}, \forall \mathrm{c}^{\prime} \subseteq \mathrm{B}, \forall \mathrm{e} \in \mathrm{E}\left[\mathrm{c}\left[\mathrm{e}>\mathrm{c}^{\prime} \Rightarrow \mathrm{c}^{\prime} \in \mathrm{C}_{\Sigma}\right]\right.$.

We denote as LEN systems the labelled EN systems in which the events represent actions which can be observable. Formally, a LEN system is a 5-tuple $\Sigma=$ $\left(\mathrm{B}, \mathrm{E}, \mathrm{F}, \mathrm{c}_{\mathrm{in}}, \mathrm{h}\right)$, where $\left(\mathrm{B}, \mathrm{E}, \mathrm{F}, \mathrm{c}_{\mathrm{in}}\right)$ is a contact free EN system (i.e. such that $\forall \mathrm{e} \in \mathrm{E}$, $\left.\forall \mathrm{c} \in \mathrm{C}_{\Sigma} \mid \cdot \mathrm{e} \subseteq \mathrm{c} \Rightarrow \mathrm{e} \bullet \cap \mathrm{c}=\varnothing \mathrm{l}\right)$ whose underlying net is such that $\mathrm{E} \subseteq \operatorname{dom}(\mathrm{F}) \cap$ $\operatorname{ran}(F)$, and $h: E \rightarrow L \cup\{\tau\}$ is the labelling function ( $\mathrm{L}$ is the alphabet of observable actions and $\tau \notin \mathrm{L}$ denotes the unobservable action).

Let $\varepsilon$ be the empty word of $E^{*}$ and $\lambda$ the empty word of $L^{*} . h$ is extended to an homomorphism $\mathrm{h}^{*}: \mathrm{E}^{*} \rightarrow \mathrm{L}^{*}$ in the following way: $\mathrm{h}^{*}(\varepsilon)=\lambda$, and $\forall \mathrm{e} \in \mathrm{E}, \forall \mathrm{w} \in$ $\left.E^{*} \mid h(e) \neq \tau \Rightarrow h^{*}(e w)=h(e) h^{*}(w) \wedge h(e)=\tau \Rightarrow h^{*}(e w)=h^{*}(w)\right]$. In the following, we denote $h^{*}$ by $h$.

Given a LEN system $\Sigma=\left(\mathrm{B}, \mathrm{E}, \mathrm{F}, \mathrm{c}_{\mathrm{in}}, \mathrm{h}\right), \quad v \in \mathrm{L}^{*}$ is enabled at the case $\mathrm{c}$, denoted $c\left(v>\right.$, iff $\left.\exists w \in E^{*}|h(w)=v \wedge c| w>\right]$. If $v$ is enabled at $c$, then the occurrence of $v$ can lead from $c$ to $c^{\prime}$, denoted $c\left(v>c^{\prime}\right.$, iff $\exists w \in E^{*}\left[h(w)=v \wedge c\left[w>c^{\prime}\right]\right.$.

According with the assumption of disregarding data and focusing on basic behaviour, we consider labelled EN systems, observing actions and states.

For what concern action observability, we associate with a class a LEN system $\Sigma=$ (B, E, F, $c_{\text {in }}, h$ ), where the labelling function $h$ associates with each event an exported class method belonging to $\mathrm{L}$. The internal methods are labelled by $\tau$.

Now we give some basic definitions based on action observability.

\section{Definition 1 (traces)}

Let $\Sigma=\left(\mathrm{B}, \mathrm{E}, \mathrm{F}, \mathrm{c}_{\mathrm{in}}, \mathrm{h}\right)$ be a LEN system. Then traces $(\Sigma)=\left\{\mathrm{w} \in \mathrm{L}^{*} \mid \mathrm{c}_{\mathrm{in}}(\mathrm{w}>\}\right.$ (i.e.: the traces are the sequences of requests that an object can accept).

\section{Definition 2 (failures)}

Let $\Sigma=\left(\mathrm{B}, \mathrm{E}, \mathrm{F}, \mathrm{c}_{\mathrm{in}}, \mathrm{h}\right)$ be a LEN system. Then failures $(\Sigma)=\left\{(\mathrm{w}, \mathrm{X}), \mathrm{w} \in \mathrm{L}^{*}, \mathrm{X}\right.$ $\subseteq \mathrm{L}|\exists \mathrm{c}| \mathrm{c}_{\mathrm{in}}(\mathrm{w}>\mathrm{c} \forall \mathrm{a} \in \mathrm{X} \underline{\operatorname{not}}(\mathrm{c}(\mathrm{a}>))\}$. 
Definition 3 (relative failures)

Let $\Sigma_{1}=\left(B_{1}, E_{1}, F_{1}, c_{i n 1}, h_{1}\right)$ and $\Sigma_{2}=\left(B_{2}, E_{2}, F_{2}, c_{i n 2}, h_{2}\right)$ be LEN systems. The set of the failures of $\Sigma_{1}$ relative to $\Sigma_{2}$ is defined as:

failures $\Sigma_{2}\left(\Sigma_{1}\right)=\left\{(\mathrm{w}, \mathrm{X}) \in\right.$ failures $\left.\left(\Sigma_{1}\right) \mid \mathrm{w} \in \operatorname{traces}\left(\Sigma_{2}\right)\right\}$.

Now we give the definition of Nierstrasz's Request substitutability preorder [11].

Definition 4 (Request Substitutabilty preorder)

Let $\Sigma_{1}=\left(B_{1}, E_{1}, F_{1}, c_{i n 1}, h_{1}\right)$ and $\Sigma_{2}=\left(B_{2}, E_{2}, F_{2}, c_{i n 2}, h_{2}\right)$ be LEN systems. $\Sigma_{1} s_{\text {RS }} \Sigma_{2}$ if and only if

i) traces $\left(\Sigma_{2}\right) \subseteq$ traces $\left(\Sigma_{1}\right)$

ii) failures $\Sigma_{2}\left(\Sigma_{1}\right) \subseteq$ failures $\left(\Sigma_{2}\right)$.

The encapsulation operator $\delta_{\mathrm{H}}[1]$ removes some transitions from a LEN system. Formally, given a LEN system $\Sigma=\left(\mathrm{B}, \mathrm{E}, \mathrm{F}, \mathrm{c}_{\mathrm{in}}, \mathrm{h}\right)$, for each $\mathrm{H} \subseteq \mathrm{L}, \delta_{\mathrm{H}}(\Sigma)=\Sigma^{\prime}$ such that $\Sigma^{\prime}=\left(B, E^{\prime}, F^{\prime}, c_{i n}, h^{\prime}\right), E^{\prime}=\{e \in E \mid h(e) \notin H\}, F^{\prime}=F \cap\left(\left(B \times E^{\prime}\right) \cup\left(E^{\prime} \times B\right)\right)$ and $h^{\prime}$ $=\mathrm{h} \cap\left(\mathrm{E}^{\prime} \times \mathrm{L}\right)$.

The abstraction operator $\tau_{\mathrm{I}}$ labels as not observable some transitions of a LEN system. Formally, given a LEN system $\Sigma=\left(\mathrm{B}, \mathrm{E}, \mathrm{F}, \mathrm{c}_{\mathrm{in}}, \mathrm{h}\right)$, for each $\mathrm{I} \subseteq \mathrm{L} \tau_{\mathrm{I}}(\Sigma)=\Sigma^{\prime}$ such that $\Sigma^{\prime}=\left(B, E, F, c_{i n}, h^{\prime}\right)$, and, for each $e \in E, h(e) \in I \Rightarrow h^{\prime}(e)=\tau$ and $h(e) \notin I$ $\Rightarrow h^{\prime}(e)=h(e)$.

Now we give some basic definitions based on state observability [14].

A pair $(\Sigma, \mathrm{O})$ (also denoted with $\Sigma$ ) is an S-labelled system iff $\Sigma=\left(\mathrm{B}, \mathrm{E}, \mathrm{F}, \mathrm{c}_{\mathrm{in}}\right)$ is an $E N$ system, $O \subseteq B$ is such that $\forall s \in O \exists \mathrm{c}$ belonging to the set of cases $C_{\Sigma}$ such that $\mathrm{s} \in \mathrm{c}$ and $\mathrm{c} \subseteq \mathrm{O}$ ( $\mathrm{O}$ is the set of the observable places, or conditions), and $\mathrm{c}_{\text {in }} \subseteq$ O. $\mathrm{C}_{\mathrm{obs}}(\Sigma)=\left\{c \in \mathrm{C}_{\Sigma}: \mathrm{c} \subseteq \mathrm{O}\right\}$ is the set of observable cases. For all $\mathrm{w} \in \mathrm{E}^{*}$, the set of firable permutation, denoted $F-\operatorname{Perm}(w)$, is the set of permutations $\mathrm{w}^{\prime}$ of $w$ such that $\exists c, c^{\prime} \in C_{\Sigma}: \quad c \mid w^{\prime}>c^{\prime}$. Let $\Sigma$ be an $S$-labelled system, w $\in E^{+}$and $c, c^{\prime} \in$ $\mathrm{C}_{\text {obs }}(\Sigma)$ such that $\mathrm{c}\left[\mathrm{w}>\mathrm{c}^{\prime}\right.$; then $\mathrm{w}$ is an elementary observable path from $\mathrm{c}$ to $\mathrm{c}^{\prime}$ iff $\forall \mathrm{u}_{1}, \ldots, \mathrm{u}_{\mathrm{k}} \in \mathrm{E}\left[\mathrm{u}_{1} \ldots \mathrm{u}_{\mathrm{k}} \in \mathrm{F}-\operatorname{Perm}(\mathrm{w}) \wedge \mathrm{c}\left[\mathrm{u}_{1}>\mathrm{c}_{1} \ldots \mathrm{c}_{\mathrm{k}-1} \mid \mathrm{u}_{\mathrm{k}}>\mathrm{c}^{\prime}\right] \Rightarrow \forall \mathrm{i}=1, \ldots, \mathrm{k}-1\right.$ $\mathrm{c}_{\mathrm{i}} \notin \mathrm{C}_{\text {obs }}(\Sigma)$.

$\mathrm{W}_{\Sigma}$ denotes the set of elementary observable paths.

Given an S-labelled system $(\Sigma, \mathrm{O})$, its observable local state transformation algebra (OLST algebra), denoted $\mathrm{I}$, is the tuple $<\mathrm{I},-, \cap, \cup, \mathrm{c}_{\mathrm{in}}, \rightarrow>$, where $\langle\mathrm{I},-, \cap, \cup\rangle$ is the observable local state algebra, where $I$ is the smallest set contained in the power set of $\mathrm{O}$ that contains $\mathrm{C}_{\mathrm{obs}}(\Sigma)$ and that is closed under the operation of set difference - ; $\rightarrow \subseteq \mathrm{I} \times \mathrm{I}$ is an unreflexive relation called observable local state transformation relation and defined as: $\forall \mathrm{x}, \mathrm{y} \in \mathrm{I} \mathrm{x} \rightarrow \mathrm{y} \Leftrightarrow \exists \mathrm{w} \in \mathrm{W}_{\Sigma}[\bullet \mathrm{w} \subseteq \mathrm{x} \wedge \mathrm{y}=(\mathrm{x}-\bullet \mathrm{w}) \cup$ $\mathrm{w} \bullet \mathrm{I}$, where $\bullet \mathrm{w}$ and $\mathrm{w} \bullet$ are respectively the pre-set and the post-set of $\mathrm{w}$, i.e.: the minimal set of conditions giving concession to the word $w$ and the set of conditions which become true after the occurrence of the word $w$.

Now we give the definition of the State Transformation preorder $\left(s_{\mathrm{ST}}\right)$ [14], which compares systems by observing local states and is such that $\Sigma_{1} s_{\mathrm{ST}} \Sigma_{2}$ if and only if the state space of $\Sigma_{1}$ is a substructure of the state space of $\Sigma_{2}$ such that for any observable local state transformation in $\Sigma_{1}$ there is a corresponding observable local state transformation in $\Sigma_{2}$.

\section{Definition 5 (State Transformation preorder)}

Let $\Sigma_{1}$ and $\Sigma_{2}$ be two S-labelled systems, $\mathbf{I}_{1}=\left\langle\mathrm{I}_{1},-, \cap, \cup, \mathrm{c}_{\mathrm{in1}}, \rightarrow_{1}>\right.$ and $\mathbf{I}_{2}=$ $<\mathrm{I}_{2},-, \cap, \cup, \mathrm{c}_{\mathrm{in} 2}, \rightarrow_{2}>$ be the respective OLST algebras. Then $\Sigma_{1} \leq_{\mathrm{ST}} \Sigma_{2}$ if and 
only if there exists an injective morphism $\mathrm{h}:<\mathrm{I}_{1},-, \cap, U>\rightarrow<\mathrm{I}_{2},-, \cap, \cup>$ such that:

i) $\mathrm{h}\left(\mathrm{c}_{\mathrm{in} 1}\right) \subseteq \mathrm{c}_{\mathrm{in} 2}$

ii) $\forall \mathrm{x}, \mathrm{y} \in \mathrm{I}_{1}\left[\mathrm{x} \rightarrow_{1} \mathrm{y} \Rightarrow\left[\mathrm{h}(\mathrm{x}) \rightarrow_{2} \mathrm{~h}(\mathrm{y}) \quad \mathrm{v}\right.\right.$

$\left.\exists \mathrm{n} \geq 1, \exists \mathrm{i}_{1}, \mathrm{i}_{2}, \ldots, \mathrm{i}_{\mathrm{n}} \in\left(\mathrm{I}_{2}-\mathrm{h}\left(\mathrm{I}_{1}\right)\right) \mathrm{h}(\mathrm{x}) \rightarrow_{2} \mathrm{i}_{1} \rightarrow_{2} \ldots \mathrm{i}_{\mathrm{n}} \rightarrow_{2} \mathrm{~h}(\mathrm{y})\right] 1$.

When the condition [ $\mathrm{x} \rightarrow_{1} \mathrm{y} \Rightarrow \mathrm{h}(\mathrm{x}) \rightarrow_{2} \mathrm{~h}(\mathrm{y})$ ] is satisfied, then the S-labelled system $\Sigma_{2}$ is an extension of $\Sigma_{1}$, while when the condition $[\mathrm{x} \rightarrow, \mathrm{y} \Rightarrow \exists \mathrm{n} \geq 1$, $\exists \mathrm{i}_{1}, \mathrm{i}_{2}, \ldots, \mathrm{i}_{\mathrm{n}} \in\left(\mathrm{I}_{2}-\mathrm{h}\left(\mathrm{I}_{1}\right)\right) \mathrm{h}(\mathrm{x}) \rightarrow_{2} \mathrm{i}_{1} \rightarrow_{2} \ldots \mathrm{i}_{\mathrm{n}} \rightarrow_{2} \mathrm{~h}(\mathrm{y}) \mathrm{l}$ is satisfied, then the S-labelled system $\Sigma_{2}$ is an expansion of $\Sigma_{1}$.

\section{Example 1}

Let us consider the systems given in figure 1, where shaded places are the observable ones, and let us consider mappings of observable places which are represented by using the same name for corresponding places. Then, the net systems $\Sigma_{1}$ and $\Sigma_{2}$ are ST-equivalent, since $\Sigma_{1} s_{\mathrm{ST}} \Sigma_{2}$ and $\Sigma_{2} s_{\mathrm{ST}} \Sigma_{1}$. Analogously, the systems $\Sigma_{4}$ and $\Sigma_{5}$ are equivalent.

$\Sigma_{2} s_{\mathrm{ST}} \Sigma_{3}$, in fact $\Sigma_{3}$ performs the analogous stāte-transformation from $\mathrm{p}_{1}$ to $\mathrm{p}_{2}$ as $\Sigma_{2}$ does, and, moreover, performs a further state transformation from $\mathrm{p}_{2}$ to $\mathrm{p}_{3}$.

Analogously, $\Sigma_{3} s_{\mathrm{ST}} \Sigma_{4}$ as $\Sigma_{4}$ extends the state transformations of $\Sigma_{3}$ by adding a branch.

In this figure, considering the S-labelled systems $\Sigma_{1}$ and $\Sigma_{3}$, where $\Sigma_{1} s_{S T} \Sigma_{3}$, if we construct the morphism $h$ that associates pl (of $\Sigma_{1}$ ) with pl (of $\Sigma_{3}$ ) and p2 (of $\Sigma_{1}$ ) with p2 (of $\Sigma_{3}$ ), then $\Sigma_{3}$ is an extension of $\Sigma_{1}$; while, if we construct the morphism $\mathrm{k}$ that associates p1 (of $\Sigma_{1}$ ) with p1 (of $\Sigma_{3}$ ) and p2 (of $\Sigma_{1}$ ) with p3 (of $\Sigma_{3}$ ), then $\Sigma_{3}$ is an expansion of $\Sigma_{1}$.

$$
\Sigma_{1} \cong_{\mathrm{ST}} \Sigma_{2} \leq_{\mathrm{ST}} \quad \Sigma_{3} \quad \leq_{\mathrm{ST}} \quad \Sigma_{4} \quad \cong_{\mathrm{ST}} \quad \Sigma_{5}
$$
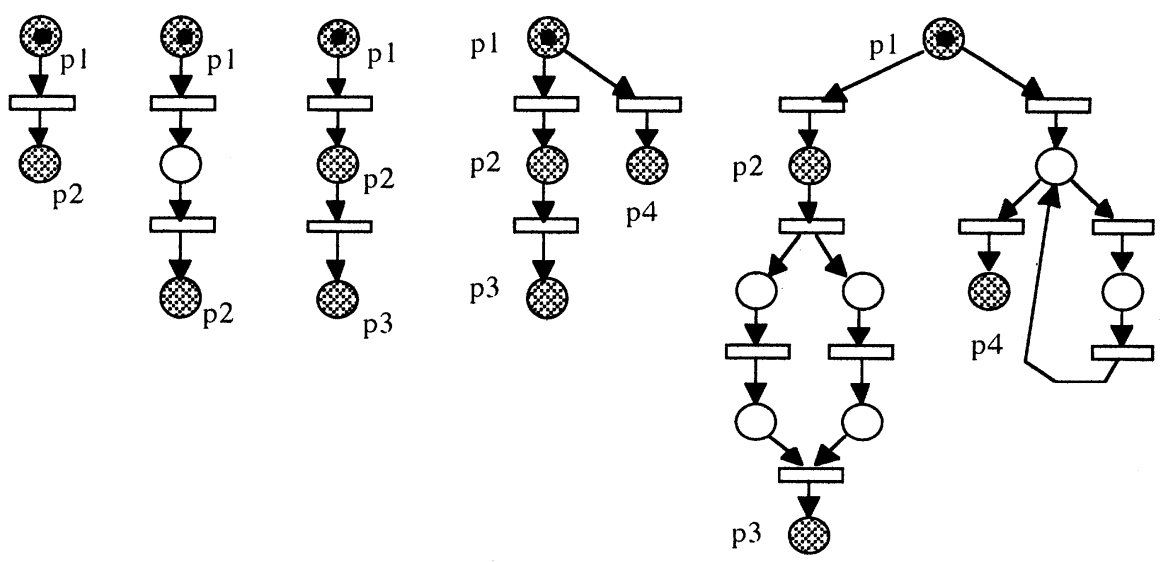

Figure 1 (Example 1). State transformation preorders and equivalences. 


\section{Examples and open problems}

Our aim here is to stress, through examples, the two different aspects and uses of inheritance relation, i.e.: the is- $a$ relation as subtyping and the implementation inheritance as code reuse. In the literature inheritance has been formalized without a clear distinction between these two forms.

In the first example, we consider Nierstrasz's RS-preorder, based on action observability, where a class $B$ is subclass of a class $A$ iff each sequence of requests accepted by $\mathrm{A}$ is also accepted by $\mathrm{B}$, and, if the class $\mathrm{A}$, after having received a sequence $s$, can receive another request $r$, then also $\mathrm{B}$, after the sequence $s$, can accept the request $r$.

In the second example, we consider the ST-preorder, based on state observability, where a class B is subclass of a class A iff the S-labelled system associated with B is an extension and/or an expansion of the S-labelled system associated with $\mathrm{A}$.

In both cases, the examples exhibit some counter-intuitive feature, that we point out.

\section{Example 2}

This example is taken from Nierstrasz's paper [11]. In figure 2, the class VAR, that represents variables, is subclass of the class BUF, that represents one place buffers. Rephrasing Nierstrasz from CCS to Petri nets, the RS-preorder between the LEN system associated with the class VAR and the LEN system associated with the class BUF is satisfied, i.e. $\Sigma_{\mathrm{VAR}} \varsigma_{\mathrm{RS}} \Sigma_{\mathrm{BUF}}$.

However, we believe that this class hierarchy is somehow misleading: while, according to Nierstrasz, a variable is seen as a (specialization of a) buffer, programmers intuition is that a buffer can be implemented through a variable. Then we want to relate the class BUF and the class VAR through a notion of implementation inheritance rather then through an is-a relation.

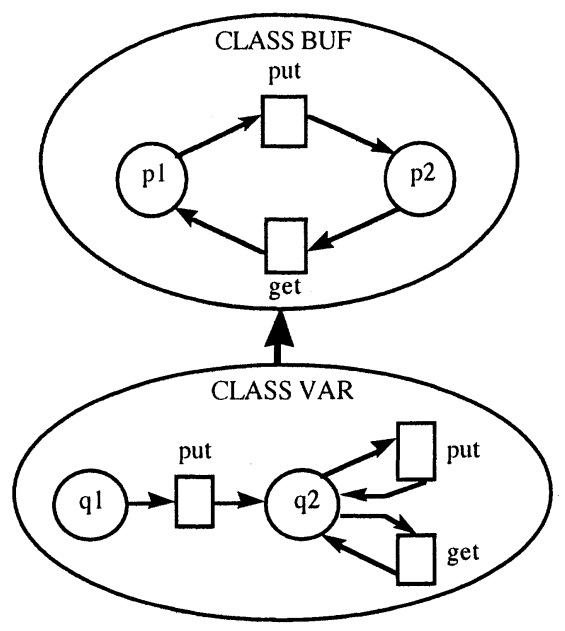

Figure 2 (Example 2). Nierstrasz's example. 


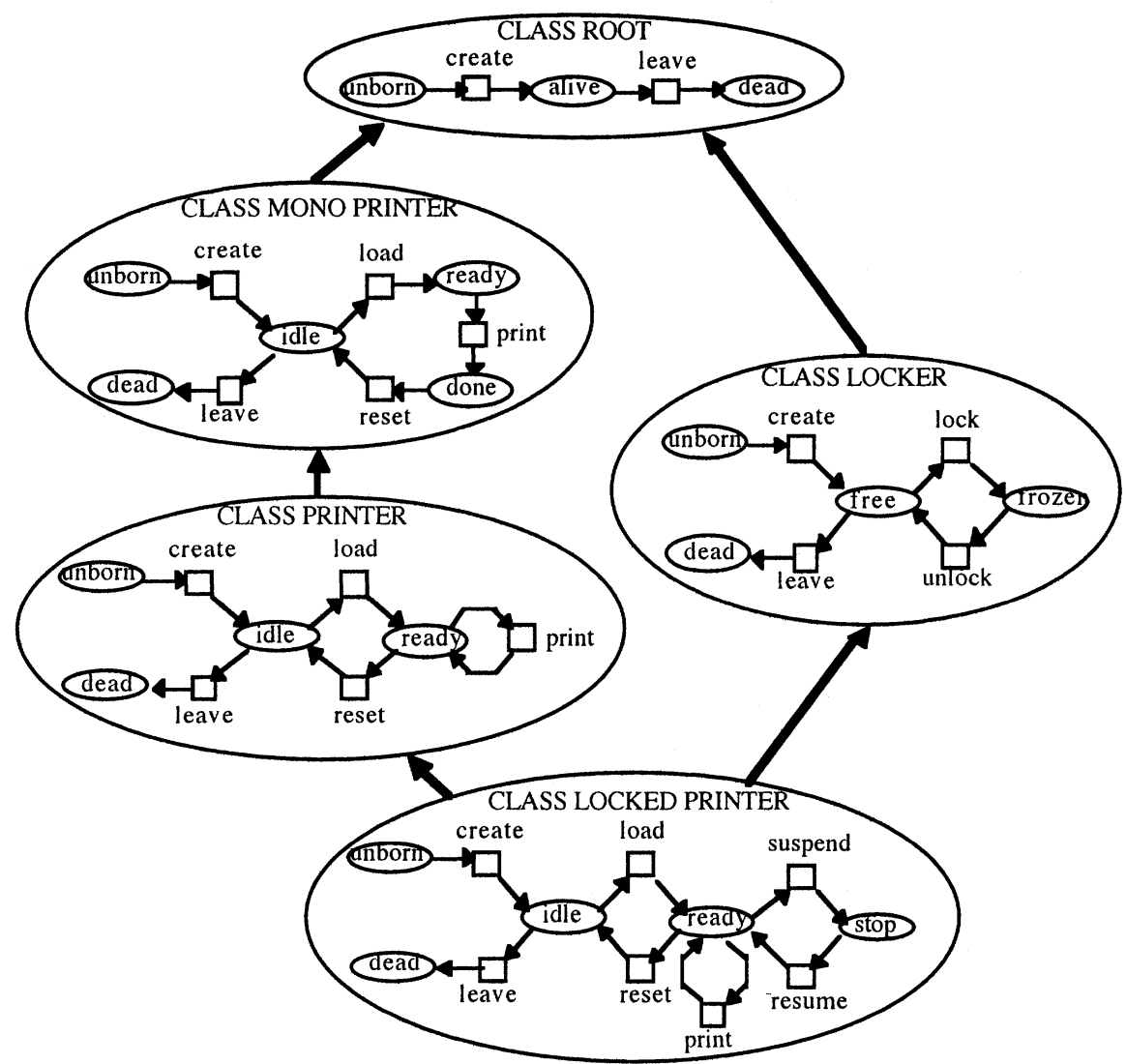

Figure 3 (Example 3). CLOWN's multiple inheritance hierarchy between printers.

\section{Example 3}

This example is taken from [5]. Figure 3 shows a multiple inheritance hierarchy between printers. In CLOWN, this hierarchy is studied through the ST-preorder, which considers the state (place) observation. However, observing states is somehow counter-intuitive: in fact, e.g., the class LOCKED PRINTER is subclass of PRINTER since it inherits the methods "load", "print" and "reset", and is subclass of LOCKER as it inherits the methods "lock" and "unlock". This is to say that one could wish to build that hierarchy observing methods (actions) rather than states. By observing states, it is possible to capture and compare the internal structure of objects, while in the hierarchy in the figure behaviour comparison requires action observation.

Indeed, the state labelling policy defined in [5] considers the state observability with respect to the exportability of methods associated with transitions. Then, the transition observability is indirectly considered.

Unfortunately, using Nierstasz's RS-preorder one can't get the previous hierarchy, since the class LOCKED PRINTER isn't subclass of LOCKER. 
Also according to van der Aalst and Bastens's approach, that combines the branching bismulation equivalence with the encapsulation and abstraction operator (see [1]), the class PRINTER can't be a subclass of the class MONO PRINTER. The life-cycle inheritance defined by van der Aalst and Basten is in fact a too strong relation, that can't be applied in many cases.

\section{The preorders based on action observability}

As said in the introduction, type inheritance concerns and compare the external observable behaviour of objects; implementation inheritance does the same with respect to their internal structure. Moreover, we believe that preorders are better suited than equivalence notions for comparing behaviour.

Therefore, we base the type inheritance on an action observation preorder, while we base implementation inheritance on a state observation preorder. In particular, the first preorder is based on failure, since bisimulation distinguishes systems also with respect to some aspect of their internal structure; in the second case we consider ST-preorder.

While this latter does not require modifications w.r.t. the original definition, we need to integrate the two approaches by Nierstrasz and van der Aalst and Basten, since no one of them is fully satisfactory, as shown by the examples in the previous section. To this purpose we define a further operator, namely the renaming operator $\rho_{\mathrm{R}, \mathrm{S}}$, and new preorders based on action observation.

Formally, for each $R \subseteq L$ and for each set of labels $S$ such that $S \cap L=\varnothing$ and $|S|=$ $|R|$, we define a bijective function $s: R \rightarrow S$, that maps each element of $\mathrm{R}$ into a new label. Let $L^{\prime}$ be a superset of $S$ containing $L-R$, i.e. $S \subseteq L^{\prime}$ and $L-R \subseteq L^{\prime}$. On the basis of the function $s$ we define the renaming function:

$r: \mathrm{L} \rightarrow \mathrm{L}^{\prime}$ that maps each label into a new label in the following way:

(1) $\quad r(\mathrm{x})=\mathrm{x}$ if $x \notin \mathrm{R}$

(2) $\quad r(\mathrm{x})=\mathrm{y}$ if $x \in \mathrm{R}, y \in \mathrm{S}$ and $s(\mathrm{x})=\mathrm{y}$.

This function $r$ is injective, since the function $s$ is bijective.

We define the renaming operator $\rho_{\mathrm{R}, \mathrm{S}}$ in the following way:

$\rho_{\mathrm{R}, \mathrm{S}}(\Sigma)=\Sigma^{\prime}$ such that $\Sigma^{\prime}=\left(\mathrm{B}, \mathrm{E}, \mathrm{F}, \mathrm{c}_{\mathrm{in}}, \mathrm{h}^{\prime}\right)$, where $\mathrm{h}^{\prime}=r \circ \mathrm{h}$.

The renaming function $r$ is extended in the following way: $r * \mathrm{~L}^{*} \rightarrow \mathrm{L}^{*}$, such that, for each $w \in L^{*}$ and for each $w^{\prime} \in L^{\prime *}, w=v_{1} v_{2} \ldots v_{n}, w^{\prime}=v^{\prime}{ }_{1} v^{\prime}{ }_{2} \ldots v_{n}^{\prime}, \quad r *(w)=$ $\mathrm{w}^{\prime}$ iff $r\left(\mathrm{v}_{1}\right)=\mathrm{v}_{1}^{\prime}, r\left(\mathrm{v}_{2}\right)=\mathrm{v}_{2}^{\prime}, \ldots r\left(\mathrm{v}_{\mathrm{n}}\right)=\mathrm{v}_{\mathrm{n}}^{\prime}$.

Now we can give the definitions of four preorders based on Nierstrasz's RS-preorder and on the encapsulation, abstraction and renaming operators. In the following, $\Sigma_{\mathrm{A}}$ and $\Sigma_{B}$ are $L E N$ systems associated with a class $A$ and a class $B$, while $L_{A}$ and $L_{B}$ are the sets of event labels associated respectively with $\Sigma_{\mathrm{A}}$ and $\Sigma_{\mathrm{B}}$ and corresponding to the methods of the classes A and B.

The first preorder, the strong substitutability, is equivalent to the RS-preoder.

\section{Definition 6}

$\Sigma_{\mathrm{B}}$ is less or equal to $\Sigma_{\mathrm{A}}$ w.r.t. strong substitutability, denoted $\Sigma_{\mathrm{B}} \varsigma_{\mathrm{SS}} \Sigma_{\mathrm{A}}$, if and only if there exists a set $\mathrm{H} \subseteq \mathrm{L}_{\mathrm{B}}$ such that $\delta_{\mathrm{H}}\left(\Sigma_{\mathrm{B}}\right){s_{\mathrm{RS}}}_{\Sigma_{\mathrm{A}}}$.

If $\Sigma_{B} s_{S S} \Sigma_{A}$ then an object of class A can be substituted by an object of class B and the environment will not be able to notice the difference, i.e.: if an object of class 
A may accept after a request sequence $w$ another request $a$, then an object of class $B$ must accept the request a after the request sequence $w$, whatever is the reached state.

The new methods added in class B are considered as not available through the encapsulation operator $\delta_{\mathrm{H}}$.

\section{Example 4}

In figure $3, \Sigma_{\text {MONO PRINTER }} \leq_{S S} \Sigma_{\text {ROOT }}, \Sigma_{\text {LOCKER }} \leq_{S S} \Sigma_{\text {ROOT }}, \Sigma_{\text {PRINTER }} s_{\text {SS }}$ $\Sigma_{\text {MONO PRINTER }}, \Sigma_{\text {LOCED PRINTER }} s_{\text {SS }} \Sigma_{\text {PRINTER }}$, but nOt $\left(\Sigma_{\text {LOCKED PRINTER }} s_{S S} \Sigma_{\text {LOCKER }}\right)$. In fact, the methods "load" and "reset" are not inherited from the class LOCKER, and if we apply the encapsulation operator to the corresponding transitions, the transitions corresponding to the methods "suspend" and "resume", inherited (and renamed) from LOCKER, become unreachable.

The second preorder, the strong substitutability with renaming, is less restrictive than strong substitutability.

\section{Definition 7}

$\Sigma_{B}$ is less or equal to $\Sigma_{A}$ w.r.t. strong substitutability with renaming, denoted $\Sigma_{\mathrm{B}} \leq_{\mathrm{SSR}} \Sigma_{\mathrm{A}}$, if and only if there exist a set $\mathrm{H} \subseteq \mathrm{L}_{\mathrm{B}}$, a set $\mathrm{R} \subseteq \mathrm{L}_{\mathrm{A}}$ and a set $\mathrm{S} \subseteq \mathrm{L}_{\mathrm{B}}$ such that $\mathrm{S} \cap \mathrm{L}_{\mathrm{A}}=\varnothing$ and $\delta_{\mathrm{H}}\left(\Sigma_{\mathrm{B}}\right) \varsigma_{\mathrm{RS}} \rho_{\mathrm{R}, \mathrm{S}}\left(\Sigma_{\mathrm{A}}\right)$.

If $\Sigma_{B} s_{S S R} \Sigma_{A}$, then an object of class A can be substituted by an object of class B but the environment must make allowance for class $A$ methods that are renamed in class $B$ by the renaming operator $\rho_{R, S}$.

The third preorder, the weak substitutability, is another extension of the strong substitutability.

\section{Definition 8}

$\Sigma_{\mathrm{B}}$ is less or equal to $\Sigma_{\mathrm{A}}$ w.r.t. weak substitutability, denoted $\Sigma_{\mathrm{B}} \boldsymbol{s}_{\mathrm{WS}} \Sigma_{\mathrm{A}}$, if and only if there exist a set $H \subseteq L_{B}$ and a set $I \subseteq L_{B}$ such that :

$\mathrm{I} \cap \mathrm{H}=\varnothing$ and $\tau_{\mathrm{I}} \circ \delta_{\mathrm{H}}\left(\Sigma_{\mathrm{B}}\right) \mathrm{s}_{\mathrm{RS}} \Sigma_{\mathrm{A}}$.

If $\Sigma_{\mathrm{B}} \boldsymbol{s}_{\mathrm{WS}} \Sigma_{\mathrm{A}}$, then an object of class $\mathrm{A}$ can be substituted by an object of class $\mathrm{B}$ and the environment will not be able to notice the difference since the new methods added in class $B$ are either considered unobservable, through the abstraction operator $\tau_{1}$, or considered unavailable, through the encapsulation operator $\delta_{\mathrm{H}}$.

The fourth and last preorder, the weak substitutability with renaming, is the weaker preorder.

\section{Definition 9}

$\Sigma_{\mathrm{B}}$ is less or equal to $\Sigma_{\mathrm{A}}$ w.r.t. weak substitutability with renaming, denoted $\Sigma_{\mathrm{B}} \varsigma_{\mathrm{WSR}} \Sigma_{\mathrm{A}}$, if and only if there exist a set $\mathrm{H} \subseteq \mathrm{L}_{\mathrm{B}}$, a set $\mathrm{I} \subseteq \mathrm{L}_{\mathrm{B}}$, a set $\mathrm{R} \subseteq \mathrm{L}_{\mathrm{A}}$ and a set $S \subseteq L_{B}$ such that $I \cap H=\varnothing, S \cap L_{A}=\varnothing$ and $\tau_{1} \circ \delta_{H}\left(\Sigma_{B}\right) s_{R S} \rho_{R, S}\left(\Sigma_{A}\right)$.

If $\Sigma_{B} \leqslant_{W S R} \Sigma_{A}$, then an object of class A can be substituted by an object of class B and the environment must consider the renamed methods, while the new methods are either considered unobservable, through the abstraction operator $\tau_{1}$, or considered unavailable, through the encapsulation operator $\delta_{\mathrm{H}}$. 


\section{Example 5}

In figure $3, \Sigma_{\text {LOCKED PRINTER }} s_{\text {WSR }} \Sigma_{\text {LOCKER }}$. In fact, I $=\{$ load, reset $\}, R=\{$ lock, unlock $\}, \mathrm{S}=$ suspend, resume $\}, r$ (create) $=$ create, $r$ (leave) $=$ leave, $r$ (lock) $=$ suspend, $r$ (unlock) $=$ resume.

First of all, we show some properties of the RS-preoder. The RS-preorder is a congruence for the encapsulation operator $\delta_{H}$, as demonstrated in the following lemma 1 , and for the renaming operator $\rho_{R, S}$ (see lemma 2) while it isn't a congrence for the abstraction operator $\tau_{\mathrm{I}}$, as shown in the example 6 . The proofs are in $[4]$ or in the internal report $[8]$.

\section{Lemma 1}

Let $\Sigma_{1}$ and $\Sigma_{2}$ be two LEN systems such that $\Sigma_{1} s_{R S} \Sigma_{2}$. Let $L_{1}$ and $L_{2}$ be the set of labels associated respectively with $\Sigma_{1}$ and $\Sigma_{2}$. Let $\mathrm{L}=\mathrm{L}_{1} \cup \mathrm{L}_{2}$. Then, for each set $\mathrm{H} \subseteq \mathrm{L}$, it holds $\delta_{\mathrm{H}}\left(\Sigma_{1}\right) s_{\mathrm{RS}} \delta_{\mathrm{H}}\left(\Sigma_{2}\right)$.

\section{Lemma 2}

Let $\Sigma_{1}$ and $\Sigma_{2}$ be LEN systems such that $\Sigma_{1} s_{\mathrm{RS}} \Sigma_{2}$. Let $\mathrm{L}_{1}$ be the set of labels corresponding to $\Sigma_{1}$ and $\mathrm{L}_{2}$ be the set of labels corresponding to $\Sigma_{2}$. Let $\mathrm{L}=\mathrm{L}_{1} \cup \mathrm{L}_{2}$. Then, for each set $R \subseteq L$ and for each set $S$ such that $S \cap L=\varnothing$, it holds $\rho_{R, S}\left(\Sigma_{1}\right)$ $s_{R S} \rho_{R, S}\left(\Sigma_{2}\right)$.

\section{Example 6}

The RS-preorder isn't a congruence for the abstraction operator $\tau_{I}$. In figure $4, \Sigma_{1}$ $\leq_{\mathrm{RS}} \Sigma_{2}$, but, if we choose $\mathrm{I}=\{\mathrm{c}\}$, then $\underline{\operatorname{not}}\left(\tau_{\mathrm{I}}\left(\Sigma_{1}\right) \mathrm{s}_{\mathrm{RS}} \tau_{\mathrm{I}}\left(\Sigma_{2}\right)\right)$. In fact, the failure (a, $\{\mathrm{a}, \mathrm{b}\}) \in$ failures $\tau_{\mathrm{I}}\left(\Sigma_{2}\right)\left(\tau_{\mathrm{I}}\left(\Sigma_{1}\right)\right)$, but $(\mathrm{a},\{\mathrm{a}, \mathrm{b}\}) \notin$ failures $\left(\tau_{\mathrm{I}}\left(\Sigma_{2}\right)\right)$.

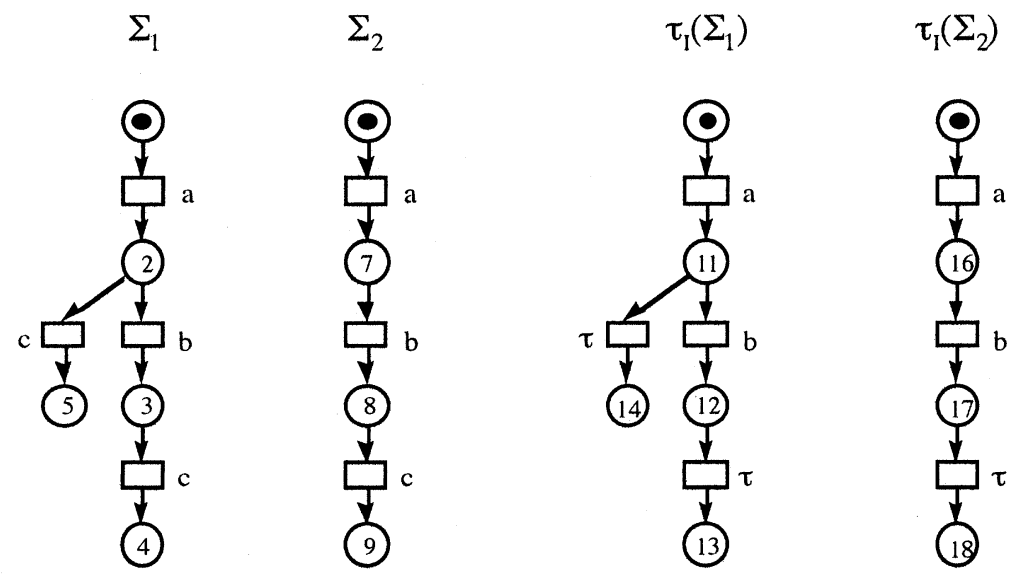

Figure 4 (Example6). RS-preorder isn't a congruence for $\tau_{1}$.

As a consequence, the abstraction operator $\tau_{1}$ must be applied to a particular set of labels I that is constructed in the following way. Let the class B be a subclass of the 
class $A$. Let $L_{B}$ and $L_{A}$ be the sets of labels corresponding to class $B$ and class $A$ methods. The abstraction operator $\tau_{I}$ is applied to a subset $B$ of class methods that are not inherited from the class $A$, i.e. $I \subseteq L_{B}-L_{A}-S$. The methods whose name is a label belonging to $\mathrm{S}$ are inherited from the class $\mathrm{A}$, then the abstraction operator can't be applied to them. The set $I$ is constructed in the following way:

(1) $I=\varnothing$, i.e. the set I is initialized to the empty set;

(2) for each $w \in$ traces $\left(\Sigma_{A}\right)$, if $w \in$ traces $\left(\Sigma_{B}\right)$ then I does't change, otherwise, let $w^{\prime} \in \operatorname{traces}\left(\Sigma_{B}\right)$ be the shorter string such that $w^{\prime}=u_{1} z_{1} u_{2} z_{2} \ldots u_{n-1} z_{n-1} u_{n}$ and $w=$ $v_{1} v_{2} \ldots v_{n-1} v_{n}$, where $v_{i} \in L_{A}^{*}, u_{i} \in L_{B}^{*}, u_{i}=r^{*}\left(v_{i}\right), i=1, \ldots, n, z_{k} \in\left(L_{B}-L_{A}-S\right) *$, $k=1, \ldots, n-1$. Then the elements of $z_{1}, z_{2}, \ldots, z_{n-1}$ are added to $I$.

Of course, if no methods of the superclass is renamed in the subclass, $S=\varnothing$ and $u_{i}=v_{i}$ for each $i=1, \ldots, n$.

On the basis of the renaming operator and of the failure equivalence we can define another equivalence relation, the failure equivalence with renaming. This definition will be usefull in order to prove the soundness of the strong substitutability with renaming and of the weak substitutability with renaming.

\section{Definition 10 (failure equivalence with renaming)}

Let $\Sigma_{1}$ and $\Sigma_{2}$ be LEN systems. $\Sigma_{1}$ is equivalent to $\Sigma_{2}$, denoted $\Sigma_{1} \approx_{\mathrm{FR}} \Sigma_{2}$, iff there exist a set $\mathrm{R} \subseteq \mathrm{L}_{2}$ and a set $\mathrm{S} \subseteq \mathrm{L}_{1}$ such that $\mathrm{S} \cap \mathrm{L}_{2}=\varnothing$ and $\Sigma_{1} \approx_{\mathrm{F}} \rho_{\mathrm{R}, \mathrm{S}}\left(\Sigma_{2}\right)$ (or $\left.\Sigma_{2} \approx_{F} \rho_{S, R}\left(\Sigma_{1}\right)\right)$.

The proof that this definition is sound is given in [4] or in [8].

The following theorem compares the four preorders.

\section{Theorem 1}

Let $\Sigma_{\mathrm{A}}$ and $\Sigma_{\mathrm{B}}$ be two LEN systems associated respectively with a class $\mathrm{A}$ and a class $B$, and let $\mathrm{L}_{A}$ and $\mathrm{L}_{B}$ be the respective sets of labels.

i) If $\Sigma_{B} s_{S S} \Sigma_{A}$, then $\Sigma_{B} s_{S S R} \Sigma_{A}$

ii) If $\Sigma_{B} s_{S S} \Sigma_{A}$, then $\Sigma_{B} s_{W S} \Sigma_{A}$

iii) If $\Sigma_{B} s_{S S R} \Sigma_{A}$, then $\Sigma_{B} s_{W S R} \Sigma_{A}$

iv) If $\Sigma_{B} \varsigma_{W S} \Sigma_{A}$, then $\Sigma_{B} \varsigma_{W S R} \Sigma_{A}$.

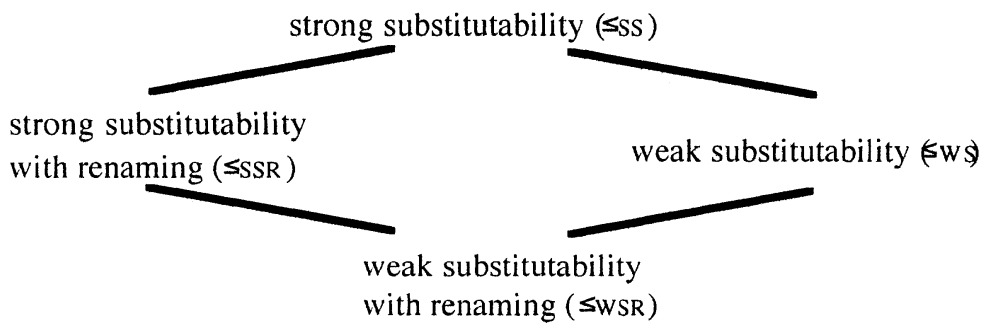

Figure 5. The relations between preorders. 


\section{Soundness of the preorders based on action observability}

In the following we demostrate that the definitions $6,7,8,9$ are sound, i.e.: that the relationships so defined are indeed preorders. The extended proofs are in [4] or in the internal report [8]; in the following we give only the main results.

\section{Theorem 2 (strong substitutability)}

The strong substitutability $\mathbf{s}_{\mathrm{SS}}$ is a preorder.

The following theorem shows that the strong substitutability is equivalent to the RS-prorder.

\section{Theorem 3}

Let $\Sigma_{1}$ and $\Sigma_{2}$ be LEN systems. Then $\Sigma_{1} s_{R S} \Sigma_{2}$ if and only if $\delta_{H}\left(\Sigma_{1}\right) s_{R S} \Sigma_{2}$, where $\mathrm{H}=\mathrm{L}_{1}-\mathrm{L}_{2}$.

In order to demonstrate that the definition 7 (strong substitutability with renaming) is sound, we must present two lemmas. The second one shows that the strong substitutability $\left(s_{S S}\right)$ is a congruence for the renaming operator $\rho_{R, S}$.

\section{Lemma 3}

Let $\Sigma$ be a LEN system and let $\mathrm{L}$ be the set of labels associated with $\Sigma$. Then for each set $\mathrm{H} \subseteq \mathrm{L}$, for each set $\mathrm{R} \subseteq \mathrm{L}$ and for each set $S$ such that $S \cap \mathrm{L}=\varnothing$, let $\rho_{\mathrm{R} S}$ a renaming operator associated with $\mathrm{R}$ and $\mathrm{S}$, and let $r: \mathrm{L} \rightarrow \mathrm{L}^{\prime}$ be the corresponding renaming function. If $\mathrm{H}^{\prime}=r(\mathrm{H})$, then $\rho_{\mathrm{R}, \mathrm{S}}{ }^{\circ} \delta_{\mathrm{H}^{\prime}}(\Sigma)=\delta_{\mathrm{H}^{\prime}}{ }^{\circ} \rho_{\mathrm{R}, \mathrm{S}}(\Sigma)$.

\section{Lemma 4}

Let $\Sigma_{1}$ and $\Sigma_{2}$ be LEN systems such that $\Sigma_{1} s_{s S} \Sigma_{2}$. Let $L_{1}$ and $L_{2}$ be the set of labels associated with $\Sigma_{1}$ and $\Sigma_{2}$ and let $\mathrm{L}=\mathrm{L}_{1} \cup \mathrm{L}_{2}$. Then, for each set $\mathrm{R} \subseteq \mathrm{L}$ and for each set $S$ such that $S \cap L=\varnothing$, it holds $\rho_{R, S}\left(\Sigma_{1}\right) s_{S S} \rho_{R, S}\left(\Sigma_{2}\right)$.

Now we can show the soundness of definition 7.

Theorem 4 (strong substitutability with renaming)

The strong substitutability with renaming $\leq_{S S R}$ is a preorder.

\section{Theorem 5 (weak substitutability)}

The weak substitutability $s_{\mathrm{ws}}$ is a preorder.

In order to demonstrate that the definition 9 (weak substitutability with renaming) is sound, we must present two lemmas. The second one shows that the weak substitutability $\left(s_{W S}\right)$ is a congruence for the renaming operator $\rho_{R, S}$.

\section{Lemma 5}

Let $\Sigma$ be a LEN system and let $L$ be the set of labels associated with $\Sigma$.

Then, for each set $\mathrm{H} \subseteq \mathrm{L}$, for each set $\mathrm{I} \subseteq \mathrm{L}$ such that $\mathrm{H} \cap \mathrm{I}=\varnothing$, for each set $\mathrm{R} \subseteq \mathrm{L}$ and for each set $\mathrm{S}$ such that $\mathrm{S} \cap \mathrm{L}=\varnothing$, let $\rho_{\mathrm{R}, \mathrm{S}}$ be a renaming operator associated with $\mathrm{R}$ and $\mathrm{S}$, and let

$r: \mathrm{L} \rightarrow \mathrm{L}^{\prime}$ be the corresponding renaming function.

If $\mathrm{H}^{\prime}=r(\mathrm{H})$ and $\mathrm{I}^{\prime}=r(\mathrm{I})$, it holds: $\rho_{\mathrm{R}, \mathrm{S}}{ }^{\circ} \tau_{\mathrm{I}}^{\circ} \delta_{\mathrm{H}}(\Sigma)=\tau_{\mathrm{I}^{\prime}}{ }^{\circ} \delta_{\mathrm{H}^{\prime}}{ }^{\circ} \rho_{\mathrm{R}, \mathrm{S}}(\Sigma)$. 


\section{Lemma 6}

Let $\Sigma_{1}$ and $\Sigma_{2}$ be LEN system such that $\Sigma_{1} s_{\text {WS }} \Sigma_{2}$. Let $L_{1}$ and $L_{2}$ be the sets of labels associated with $\Sigma_{1}$ and $\Sigma_{2}$ and let $\mathrm{L}=\mathrm{L}_{1} \cup \mathrm{L}_{2}$. Then, for each set $\mathrm{R} \subseteq \mathrm{L}$ and for each set $S$ such that $S \cap L=\varnothing$, it holds $\rho_{\mathrm{R}, S}\left(\Sigma_{1}\right) s_{\mathrm{WS}} \rho_{\mathrm{R}, S}\left(\Sigma_{2}\right)$.

Theorem 6 (weak substitutability with renaming)

The weak substitutability with renaming $s_{\mathrm{WSR}}$ is a preorder.

\section{Inheritance as subtyping and code reuse}

We now formalise the notion of type inheritance and implementation inheritance, and illustrate them on the hierarchies considered in Examples 2 and 3.

Type inheritance is based on the weak substitutability with renaming defined in the section "the preorders based on action observability" (see def. 9). A class B is a subclass of a class $\mathrm{A}$ if there is an is- $a$ relation between the subclass and the superclass. An object of class A can be substituted by an object of class B, but the environment must take into account the renamed methods and the new methods added in the subclass.

\section{Definition 11}

Let $\Sigma_{\mathrm{A}}$ and $\Sigma_{\mathrm{B}}$ be LEN systems associated with a class A and a class B. Then B is subclass of A with respect to type inheritance if and only if $\Sigma_{B} s_{W S R} \Sigma_{A}$.

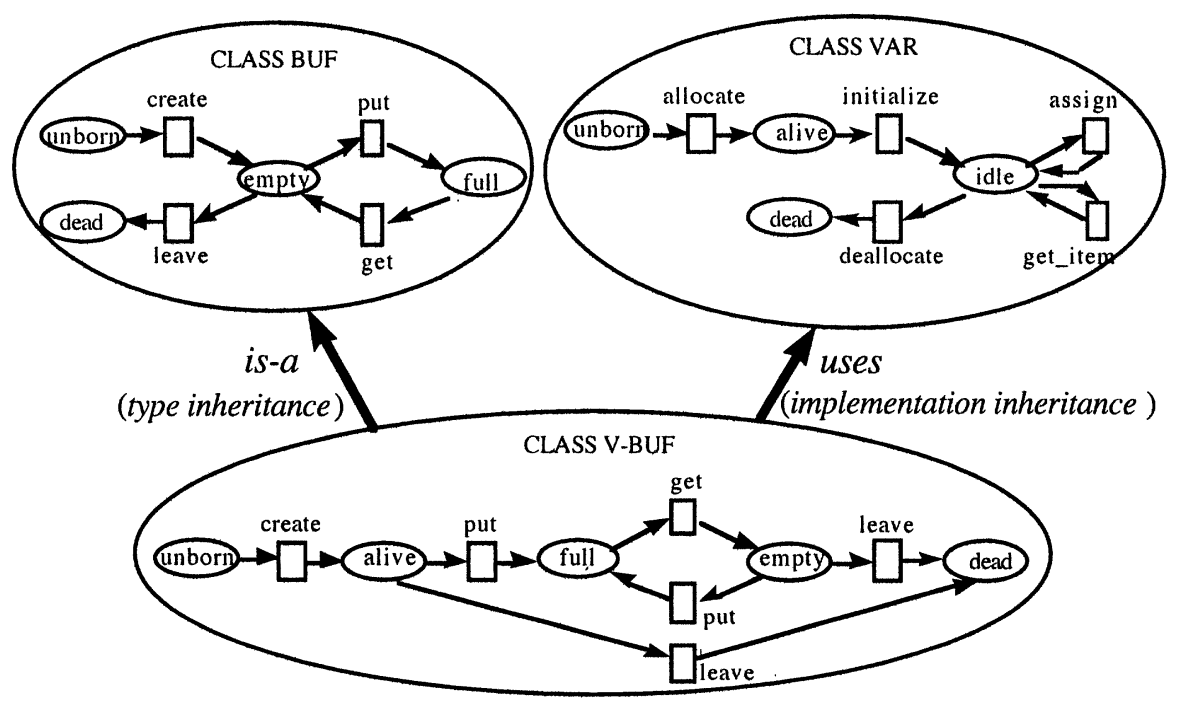

Figure 6 (Examples 7 and 8). Inheritance as subtyping and code reuse. 


\section{Example 7}

In figure 6, the net associated with the class BUF represents the behaviour of one place buffers, while the net of the class VAR represents the behaviour of variables. The net of class V-BUF represents the behaviour of one place buffers implemented through a variable, which inherits from both classes BUF and VAR. This captures programmers intuition who use variables to implement one cell buffers preserving the exhibited behaviour.

Let's verify that the corresponding definitions hold. The class V-BUF is subclass of BUF with respect to type inheritance, as it preserves the requests that can be accepted from clients. In fact, between the subclass and the superclass the strong substitutability holds: $\Sigma_{\mathrm{V} \text {-BUF }} \mathbf{s}_{\mathrm{SS}} \Sigma_{\mathrm{BUF}}$ (and then, for theorem 1, also the weak substitutability with renaming holds: $\left.\Sigma_{\mathrm{V} \text {-BUF }} s_{\mathrm{WSR}} \Sigma_{\mathrm{BUF}}\right)$. In particular, $\mathrm{H}=\varnothing$, since in the subclass every method is inherited from the superclass, then $\Sigma_{\mathrm{V} \text {-BUF }} \leq_{\mathrm{RS}} \Sigma_{\mathrm{BUF}}$. This is true, since traces $\left(\Sigma_{\mathrm{BUF}}\right) \subseteq$ traces $\left(\Sigma_{\mathrm{V} \text {-BUF }}\right)$ and failures $\Sigma_{\mathrm{BUF}}\left(\Sigma_{\mathrm{V} \text {-BUF }}\right) \subseteq$ failures $\left(\Sigma_{\mathrm{BUF}}\right)$.

Implementation inheritance is based on the State Transformation preorder (see def. 5). If a class $B$ is a subclass of a class $A$, then the system associated with $B$ must be an extension or an expansion of the system associated with class $A$, i.e. the internal structure of the class $\mathrm{B}$ is an extension or an expansion of the internal structure of the class A.

\section{Definition 12}

Let $\Sigma_{\mathrm{A}}$ and $\Sigma_{\mathrm{B}}$ be EN systems, associated with a class $\mathrm{A}$ and a class $\mathrm{B}$, in which some appropriate places are observable. Then $\mathrm{B}$ is subclass of $\mathrm{A}$ with respect to implementation inheritance if and only if $\Sigma_{\mathrm{A}} \leqslant_{\mathrm{ST}} \Sigma_{\mathrm{B}}$.

\section{Example 8}

In figure 6, we must now verify that the class V-BUF is subclass of VAR with respect to implementation inheritance: $\Sigma_{\mathrm{VAR}} s_{\mathrm{ST}} \Sigma_{\mathrm{V} \text {-BUF }}$, as the VAR methods "assign" and "get_item" are used for implementing the buffer's "put" and "get" methods.

To prove that the preorder holds, all places are considered observable. Then, the sets of observable cases are:

$\mathrm{I}_{\mathrm{VAR}}=\left\{\varnothing,\left\{\right.\right.$ unborn $\left._{\mathrm{VAR}}\right\},\left\{\right.$ alive $\left._{\mathrm{VAR}}\right\},\left\{\right.$ idle $\left.\left._{\mathrm{VAR}}\right\},\left\{\operatorname{dead}_{\mathrm{VAR}}\right\}\right\}$,

$I_{\mathrm{V}-\mathrm{BUF}}=\left\{\varnothing,\left\{\right.\right.$ unborn $\left._{\mathrm{V}-\mathrm{BUF}}\right\},\left\{\right.$ full $\left._{\mathrm{V}-\mathrm{BUF}}\right\},\left\{\right.$ empty $\left._{\mathrm{V}-\mathrm{BUF}}\right\},\left\{\right.$ idle $\left.\left._{\mathrm{V}-\mathrm{BUF}}\right\},\left\{\operatorname{dead}_{\mathrm{V}-\mathrm{BUF}}\right\}\right\}$.

The morphism $\mathrm{h}$ is constructed in the following way:

$\mathrm{h}(\varnothing)=\varnothing, \mathrm{h}\left(\left\{\right.\right.$ unborn $\left.\left._{\mathrm{VAR}}\right\}\right)=\left\{\right.$ unborn $\left._{\mathrm{V}-\mathrm{BUF}}\right\}, \mathrm{h}\left(\left\{\right.\right.$ alive $\left.\left._{\mathrm{VAR}}\right\}\right)=\left\{\right.$ alive $\left._{\mathrm{V}-\mathrm{BUF}}\right\}$,

$\mathrm{h}\left(\left\{\right.\right.$ idle $\left.\left._{\mathrm{VAR}}\right\}\right)=\left\{\right.$ full $\left._{\mathrm{V}-\mathrm{BUF}}\right\}$ (or h $\left(\left\{\right.\right.$ idle $\left.\left._{\mathrm{VAR}}\right\}\right)=\left\{\right.$ empty $\left.\left._{\mathrm{V}-\mathrm{BUF}}\right\}\right)$, $\mathrm{h}\left(\left\{\operatorname{dead}_{\mathrm{VAR}}\right\}\right)=\left\{\operatorname{dead}_{\mathrm{V}-\mathrm{BUF}}\right\}$.

The property of ST-preorder are satisfied:

(1) $\mathrm{h}\left(\left\{\right.\right.$ unborn $\left.\left._{\mathrm{VAR}}\right\}\right)=\left\{\right.$ unborn $\left._{\mathrm{V}-\mathrm{BUF}}\right\}$;

(2) $\left\{\right.$ unborn $\left._{\mathrm{VAR}}\right\} \rightarrow_{\mathrm{VAR}}\left\{\right.$ alive $\left._{\mathrm{VAR}}\right\} \Rightarrow\left\{\right.$ unborn $\left._{\mathrm{V}-\mathrm{BUF}}\right\} \rightarrow_{\mathrm{V}-\mathrm{BUF}}\left\{\right.$ alive $\left._{\mathrm{V}-\mathrm{BUF}}\right\}$,

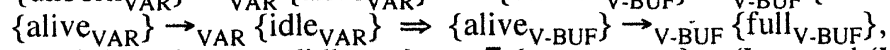
$\left\{\right.$ idle $\left._{\mathrm{VAR}}\right\} \rightarrow_{\mathrm{VAR}}\left\{\right.$ idle $\left._{\mathrm{VAR}}\right\} \Rightarrow \exists\left\{\right.$ empty $\left._{\mathrm{V}-\mathrm{BUF}}\right\} \in\left(\mathrm{I}_{\mathrm{V}-\mathrm{BUF}}-\mathrm{h}\left(\mathrm{I}_{\mathrm{VAR}}\right)\right)$ such that $\left\{\right.$ full $\left._{\mathrm{V}-\mathrm{BUF}}\right\} \rightarrow \rightarrow_{\mathrm{V}-\mathrm{BUF}}\left\{\right.$ empty $\left._{\mathrm{V}-\mathrm{BUF}}\right\} \rightarrow_{\mathrm{V}-\mathrm{BUF}}\left\{\right.$ full $\left._{\mathrm{V}-\mathrm{BUF}}\right\}$,

$\left\{\right.$ idle $\left._{\mathrm{VAR}}\right\} \rightarrow \rightarrow_{\mathrm{VAR}}\left\{\right.$ dead $\left._{\mathrm{VAR}}\right\} \stackrel{\text {-BuF }}{\Rightarrow} \exists\left\{\right.$ empty $\left._{\mathrm{V}-\mathrm{BUF}}\right\} \in\left(\mathrm{I}_{\mathrm{V}-\mathrm{BUF}}-\mathrm{h}\left(\mathrm{I}_{\mathrm{VAR}}\right)\right)$ such that $\left\{\right.$ full $\left._{\text {V-BUF }}\right\} \rightarrow_{\text {V-BUF }}\left\{\right.$ empty $\left._{\text {V-BUF }}\right\} \rightarrow_{\text {V-BUF }}\left\{\operatorname{dead}_{V-B U F}\right\}$. 


\section{Example 9}

The classes of the printer hierarchy in figure 3 satisfy the given definition. That is, the hierarchy is now captured observing methods instead of states.

In fact, as shown in the example 4 , the $s_{S S}$ relation holds for the ordered pairs (MONO PRINTER, ROOT), (LOCKER, ROOT), (PRINTER, MONO PRINTER), (LOCKED PRINTER, PRINTER). Therefore, because of theorem 1 (ii and iv), for these pairs the $\leq_{\text {WSR }}$ relation holds too. Moreover, as shown in the example $5, \Sigma_{\text {LOCKED PRINTER }}$ $s_{\text {WSR }} \Sigma_{\text {LOCKER }}$.

\section{Conclusions}

In this paper we propose preordes based on action observation for characterising type inheritance, and based on state observation for characterising implementation inheritance. To this purpose, we defined a new preorder based on action observation, namely the weak substitutability with renaming preorder, assuming Nierstrasz's hypothesis that the sequences of requests that an object can accept constitute a regular language. The result is promising as the two inheritance relations so defined support class hierarchies which look natural and appropriate to solve critical examples in the literature.

The (major) open problem is the characterisation of an object behaviour and, consequently, the definition of the inheritance relations, when the Nierstrasz's constraint is removed, i.e., when the sequences of requests that an object can accept constitute a non-regular language. In this case, the behaviour would be described by an high-level net, with guards associated with transitions. (In CLOWN, e.g., the class semantics, i.e. the object behaviour, is described by modular algebraic high level nets [6]). The problem is not at all trivial since the notions of equivalence and preorder between nets are defined in terms of EN or PT systems, i.e. low level systems, while there aren't equivalences defined for high level models. Moreover, the proposed notions of type and implementation inheritance would be embedded into a concurrent object oriented specification language: we intend to reuse CLOWN as the framework to achieve this goal.

\section{References}

11) W.M.P. van der Aalst, T. Basten, Life-Cycle Inheritance. A Petri-Net-Based Approach, in P. Azéma G. Balbo (eds.), Proc. 18th Int. Conf. on Applications and Theory of Petri Nets, LNCS 1248, Springer Verlag, 1997.

121 G. Agha, F. De Cindio, eds., Proc. of the Workshop on Object-Oriented Programming and Models of Concurrency, 1995 and 1996.

131 P. America, Issues in the Design of a Parallel Object-Oriented Language, in "Formal Aspects of Computing", vol. 1, pp. 336-411, 1989.

141 C. Balzarotti, Equivalenze all'osservazione per la caratterizzazione semantica della ereditarietà in linguaggi a oggetti concorrenti, Tesi di laurea, Università degli Studi di Milano, 1998. 
15] E. Battiston, A. Chizzoni, F. De Cindio, CLOWN as a Testbed for Concurrent Object-Oriented Concepts, G. Agha, F. De Cindio, G. Rozenberg (eds.), Advances in Petri Nets, Springer Verlag (to appear).

[6] E. Battiston, F. De Cindio, G. Mauri, Modular Algebraic Nets to Specify Concurrent Systems, IEEE Trans. on Soft. eng., vol. 22, no. 10, october 1996, pp. 689705 .

171 C. Balzarotti, F. De Cindio, L. Pomello, Observation equivalences for type and implementation inheriances, Proc. of the Workshop on Semantics of Objects as Processes, Aalborg, Denmark, 1998, in BRICS Notes Series NS-98-5, http://www.brics.dk/NS/98/5.

|8| C. Balzarotti, F. De Cindio, L. Pomello, Observation equivalences for the semantics of inheritance, DSI internal report, 1998.

19] W. Brauer, W. Reisig, G. Rozenberg, Petri Nets. Central Models and their Properties. Advances in Petri Nets 1986, part 1. Proceedings of an Advanced Course. LNCS 254, Berlin, Springer Verlag, 1987.

[10] W.R. Cook, W.L. Hill, P.S. Canning, Inheritance Is Not Subtyping, in Proc. of the ACM Symp. on Principles of Programming Languages, pp. 125-135, 1990.

[11] O. Nierstrasz, Regular Types for Active Objects, in ACM Sigplan Notices, 28(10), Proceedings of the 8th annual conference on Object-Oriented Programming Systems, Languages and Applications, OOPSLA'93, Washington DC, pp. 1-15, 1993.

[12] O. Nierstrasz, M. Papathomas, eds., Object based Concurrency and Reuse, Workshop W6, in Proc. 6th European Conf. on Object-Oriented Programming, Springer Verlag, 1992.

[13] L. Pomello, G. Rosenberg, C. Simone, A Survey of Equivalence Notions for Net Based Systems, in G. Rosenberg (ed.), "Advanced in Petri Nets", LNCS 609, SpringerVerlag, pp. 410-472, 1992.

1141 L. Pomello, C. Simone, A State Transformation Preorder over a class of EN Systems, in G. Rozenberg (ed.) APN'90, LNCS 483, pp.436-456, 1991.

115] P. Wegner, S. B. Zdonik, Inheritance as an Incremental Modification Mechanism or What Like Is and Isn't Like, in S. Gjessing and K. Nygaard (eds.), Proc.ECOOP '88, LNCS 322, Springer Verlag, pp.55-77, 1988.

This research has been supported by MURST. 